\title{
Segmentation of Hyper-Acute Ischemic Infarcts from Diffusion Weighted Imaging Based on Support Vector Machine
}

\author{
Yuqing Peng1,2, Xiaodong Zhang ${ }^{1}$, Qingmao $\mathrm{Hu}^{{ }^{*}}$ \\ ${ }^{1}$ Research Lab for Medical Imaging and Digital Surgery, Shenzhen Institutes of Advanced Technology, Chinese \\ Academy of Sciences, Shenzhen, China \\ ${ }^{2}$ Shenzhen College of Advanced Technology, University of Chinese Academy of Sciences, Shenzhen, China \\ Email: *qm.hu@siat.ac.cn
}

Received October 2015

\begin{abstract}
Accurate and automatic segmentation of hyper-acute ischemic infarct from magnetic resonance imaging is of great importance in clinical trials. Manual delineation is labor intensive, exhibits great variability due to unclear infarct boundaries, and most importantly, is not practical due to urgent time requirement for prompt therapy. In this paper, segmentation of hyper-acute ischemic infarcts from diffusion weighted imaging based on Support Vector Machine (SVM) is explored. Experiments showed that SVM could provide good agreement with manual delineations by experienced experts to achieve an average Dice coefficient of $0.763 \pm 0.121$. The proposed method could achieve significantly higher segmentation accuracy and could be a potential tool to assist clinicians for quantifying hyper-acute infarction and decision making especially for thrombolytic therapy.
\end{abstract}

Keywords

İschemic Stroke, Infarct Segmentation, Feature Selection, SVM

\section{Introduction}

Ischemic stroke remains the second cause of death worldwide with social costs increasing year by year [1]. Hyper-acute ischemic stroke is an emergency as brain tissues would be deprived of oxygen and blood supply after stroke. At hyper-acute stage diffusion weighted (DW) imaging is preferred due to its high sensitivity in detecting stroke lesions [2]. Ischemic infarcts are hard to be delineated from DW imaging on account of diverse locations, variable shapes and unclear boundaries even for experienced experts, which is especially the case for infarcts at hyper-acute stage as they are still undergoing progression and exhibiting most variations [3]. Therefore an automated and effective method is necessary to segment infarcts to aid the decision making for therapy.

Prakash et al. [3] segmented infarcts from DW images (DWIs) by first identifying axial slices with ischemic

\footnotetext{
*Corresponding author.
}

How to cite this paper: Peng, Y.Q., Zhang, X.D. and Hu, Q.M. (2015) Segmentation of Hyper-Acute Ischemic Infarcts from Diffusion Weighted Imaging Based on Support Vector Machine. Journal of Computer and Communications, 3, 152-157. 
lesions followed by binarization with a global DWI intensity threshold derived from histogram divergence. Tsai and co-workers segmented acute cerebral infarcts from DWIs and apparent diffusion coefficient (ADC) maps base on fuzzy C-means clustering (FCM) determining the histographic distribution and defining self-adjusted intensity thresholds [4].

We investigate support vector machine (SVM) on hyper-acute ischemic infarcts segmentation from DW imaging. Features are derived from DW imaging, and then carefully chosen by several feature selection methods. The infarcts are initially determined from our previous work based on hypo-intense ADC map [5], followed by refinement through training and classifying based on optimized features and SVM. To evaluate the contribution of each image, we conduct experiments through training one image and one image left out.

\section{Material and Methods}

\subsection{Datasets}

In this study, 98 ischemic stroke patients (31 women and 66 men, with age range 24 - 78 years) within 6 hours from the symptom onset were used. They were collected from Tiantan Hospital and Tianjin Huanhua Hospital since 2005. The protocol of the research has been approved by the Institutional Review Board of both hospitals. All patients gave written consent and provided permission for scientific and educational purpose. The baseline DW imaging was carried out with two 3.0 Tesla scanners (Trio-Tim, Erlagen, Germany) with a spin-echo, multislice, and single shot echo-planar of $b=0$ (B0 image) and 1000 or $1500 \mathrm{~s} / \mathrm{mm}^{2}$ (DWI) and the corresponding ADC map. The imaging covered the whole brain, with 19 - 24 axial slices of a $5 \mathrm{~mm}$ slice thickness and 1 - 1.5 mm gap, most matrixes being $128 \times 128$ with few being $156 \times 156$ or $384 \times 384$ to have in-plane resolution of $0.60 \mathrm{~mm}$ to $1.80 \mathrm{~mm}$.

\subsection{Overview of the Study}

Our study consist of the following components: extracting relevant features, employing feature selection algorithms to choose more robust features, and applying SVM to segment ischemic infarcts. The algorithm was implemented with Matlab version 2013a running on Microsoft windows with Core i5 3.2 GHz processor and 8GB memory. The schematic diagram is shown in Figure 1.

\subsection{Preprocessing}

All images were normalized to the range of [0, 255]. Asymmetry map (ASM) was calculated to reflect the characteristics of infarcts in DWI and ADC: being hyper-intense on DWI, hypo-intense on ADC, and being generally asymmetrical with respect to the midsagittal plane (MSP) extracted from B0 [6].

$$
\begin{gathered}
D W I_{-} A D C=\left\{\begin{array}{cc}
D W I-A D C, & \text { if } D W I-A D C>0 \\
0, & \text { otherwise }
\end{array}\right. \\
D I F(x, y, z)=D W I_{-} A D C(x, y, z)-\max _{(u, v) \in N_{s}\left(x_{0}, y_{0}\right)} D W I_{-} A D C(u, v, z) \\
A S M(x, y, z)=\left\{\begin{array}{cc}
D I F(x, y, z), & \text { if } D I F(x, y, z)>0 \\
0, & \text { otherwise }
\end{array}\right.
\end{gathered}
$$

where $\left(x_{0}, y_{0}\right)$ and $(x, y)$ are symmetrical with respect to the midsagittal line (which is the intersection between the MSP and the axial slice) on the axial slice $z, N_{s}\left(x_{0}, y_{0}\right)$ is the neighborhood of $\left(x_{0}, y_{0}\right)$ and the window size of window is $5 \times 5$. Axial slices of B0, DWI, ADC and ASM of a patient are shown in Figure 2. It is clear that the infarct region on the right hemisphere has been enhanced to be more easily discernable than both the DWI and ADC.

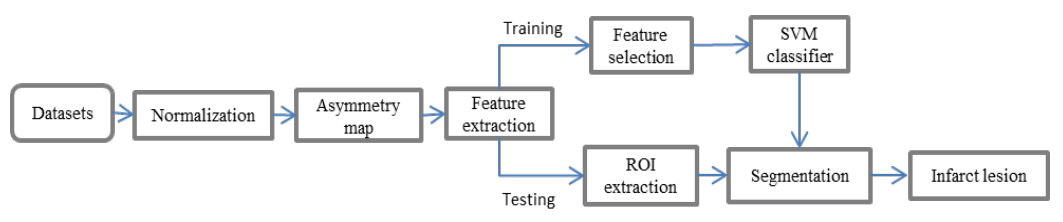

Figure 1. Flowchart of the proposed method. 

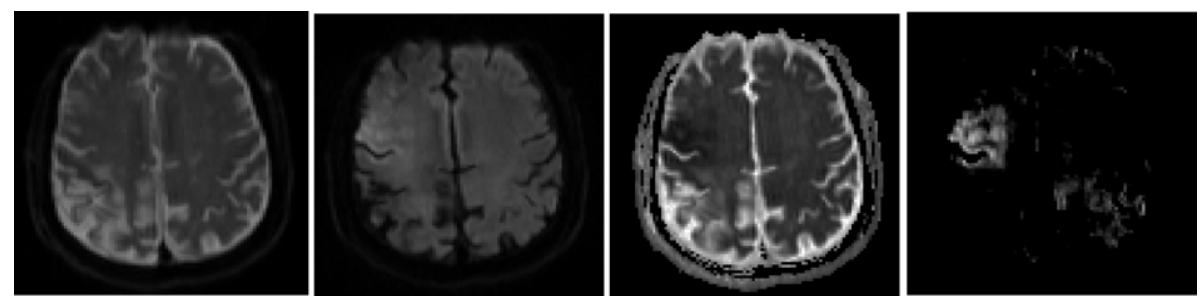

Figure 2. From left to right, axial slices of B0, DWI, ADC and ASM.

\subsection{Region of Interest}

To alleviate the interference of normal brain tissues and speed up the calculation, candidate infarcts in the form of regions of interest (ROIs) are extracted from thresholding ADC maps subjected to DWI [5]. Specifically, denote the most frequent $\mathrm{ADC}$ value for all voxels within the brain as $\mathrm{ADC}_{\mathrm{ref}}$, any voxels with $\mathrm{ADC}$ not greater than $0.75^{*} \mathrm{ADC}_{\text {ref }}$ are to formulate connected components. Any connected components with average DWI values not smaller than the intensity average plus the intensity standard deviation of brain voxels on DWI at the corresponding axial slice are kept as part of region $\mathrm{R}_{1}$. Voxels with $\mathrm{ADC}$ value within $\left(0.75 * \mathrm{ADC}_{\mathrm{ref}}, 0.85 * \mathrm{ADC}_{\mathrm{ref}}\right]$ are checked to formulate connected components and added to $\mathrm{R}_{1}$ if the components has at least one neighboring voxels in $R_{1} . R_{1}$ is then increased by morphological dilation with a structuring element of radius $R_{d}$ (being 2 voxels). Suppose the $\mathrm{z}$ coordinates of $\mathrm{R}_{1}$ are in the range of $\mathrm{z}_{0}$ to $\mathrm{z}_{\mathrm{n}}$ with $\mathrm{z}_{\mathrm{n}}>=\mathrm{z}_{0}$, then $\mathrm{R}_{1}$ region on the axial slice $z_{0}$ and $z_{n}$ are respectively pasted to axial slice $z_{0}-1$ and $z_{n}+1$ to attain the eventual ROIs. Figure 3 shows an axial slice of DWI, ADC and the corresponding ROIs (in color), where regions in red are initial ischemic infarcts and those in yellow are added voxels through the described morphological dilation.

\subsection{Feature Extraction}

For training, the voxels in infarct regions are the positive samples and drawn by a specialist. While the negative samples are those voxels close to the infarct regions obtained through iterative dilation of the infarct regions such that the number of negative samples is just equal to or greater than that of the positive samples. The features for each voxel are: intensity value, mean value, standard deviation, the weighted mean intensity value with Gaussian kernel (mean being 0 and standard deviation being 1), minimum value, maximum value, median value, skewness, kurtosis. All of these features are calculated from a local window of size $3 \times 3$ except the intensity value. The feature vector is formulated form the 9 quantities of B0, DWI, ADC and ASM to be 36 dimensional.

\subsection{Training}

Segmentation of ischemic infarcts can be considered as a classification of two classes: infarcts and non-infarcts. Two-fold cross-validation is applied in this research. The classifier is SVM while features are optimized through the feature selection procedure detailed below.

SVM was first proposed by Vapnik [7] as a supervised learning method to transform training samples into a multidimensional hyperspace and then computes decision boundary in hyperplane by solving the convex optimization problem. The margin between the positive and negative samples from the separating hyperplane is to be maximal. To obtain the maximal margin, we employ LIBSVM tool box to obtain optimal parameters [8], using radial basis kernel functions (RBFs). Two important parameters of the RBF, penalty factor $C$ and the parameter $\gamma$, are respectively set to 32 and 0.5 using grid search method to obtain a good performance for training samples.

\subsection{Feature Selection}

Redundancy and irrelevant features may lead to non-effective classification model. It is desirable to rank the importance of the features and select the distinguishing features. Five feature selection methods are employed and compared in our experiments.

Fisher score (FS) [9] identifies features with small within-class distance and large between-class distance. Threshold value is used to differentiate effective features. 


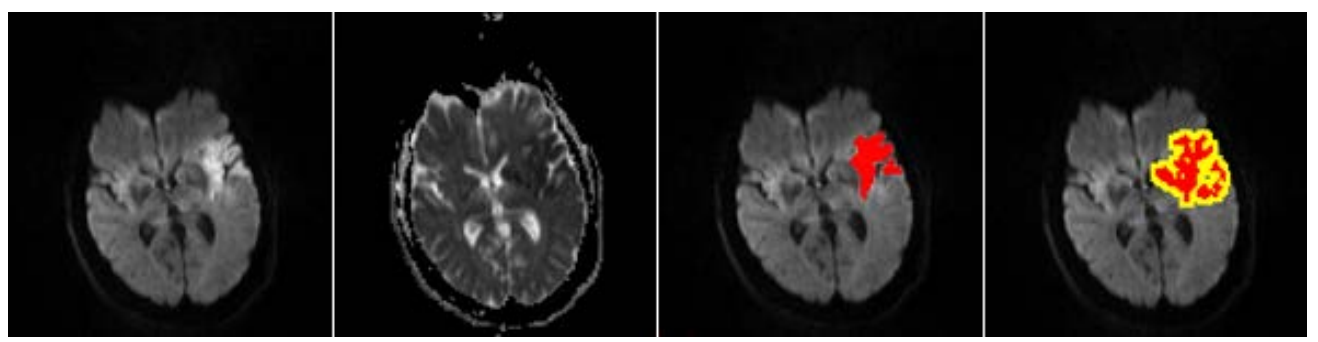

Figure 3. From left to right, an axial slice of DWI, ADC, the segmented ischemic regions form [5] on DWI and the corresponding ROIs for detecting infarction overlaid in color on DWI, where the region in red are initial ROIs from [5] while those in yellow are from morphological dilation.

Gini index (Gini) [10] quantifies the ability of feature to differentiate the classes. The features are relevant with smaller Gini Index. The top $\mathrm{k}$ features with the smallest Gini index are selected.

Mutual-information (MI) [11] describes the relationship between the features and categories. It calculates information between the feature and the category with a larger MI for larger information.

Minimum redundancy maximum relevance (mRMR) [12] is a filtering method based on space search. It searches for the feature subsets by using information gap and information entropy to obtain the feature subsets.

ReliefF algorithm [13] computes feature weight to rank features with linear time according to the number of features extracted from datasets.

\section{Results}

The proposed segmentation method has been evaluated on 2-fold cross validation. All datas are divided into two groups randomly with equal numbers.

\subsection{Performance Evaluation Metrics}

To assess the performance of the segmentation, three popular evaluation metrics are used, the Dice coefficient (Dice), sensitivity and specificity as calculated below: Dice $=2 \mathrm{TP} /(\mathrm{FP}+\mathrm{FN})$, sensitivity $=\mathrm{TP} /(\mathrm{TP}+\mathrm{FN})$, and specificity $=\mathrm{TN} /(\mathrm{TN}+\mathrm{FP})$, where $\mathrm{TP}$ is for the number of correctly segmented infarct voxels, $\mathrm{TN}$ is for the number of correctly segmented non-infarct voxels, FP is for the number of wrongly segmented infarct voxels, and FN is for the number of wrongly segmented non-infarct voxels.

\subsection{Classifiers with Different Image Combinations}

To see the relative importance of different images in the classifier, we designed classifiers with each singular image and classifiers with one image being excluded. For instance, we designed a classifier from ASM image to obtain an average Dice of $0.749 \pm 0.122$. The results are shown in Figure 4 .

\subsection{Performance of Different Feature Selection Methods}

We compared the suitability of a number of feature selection methods. Each selector is applied to the training data and returns a ranking of the feature vector. Different sizes of features are tested on each selector. The mean Dice over all testing images on SVM are shown in Figure 5.

The highest Dice is achieved from feature selection using on MI algorithm with 15 features on SVM. The mean Dice, sensitivity and specificity are $0.763 \pm 0.121,76.08 \% \pm 14.8 \%, 99.95 \% \pm 0.098 \%$ respectively. Two slices with good and bad segmentation are shown in Figure 6.

\subsection{Comparison with Existing Methods}

The proposed method has been compared with divergence measure based method (DM method) [3] and FCM based method [4]. For a fair and relevant comparison, we have tried our best to implement them to attain the best performance on the 98 datasets within 6 hours from symptom onset. The Dice, sensitivity and specificity for [3] ([4]) are respectively $0.215 \pm 0.213(0.597 \pm 0.204), 0.565 \pm 0.346(0.585 \pm 0.221)$ and $0.983 \pm 0.024(0.999$ $\pm 0.001)$. 


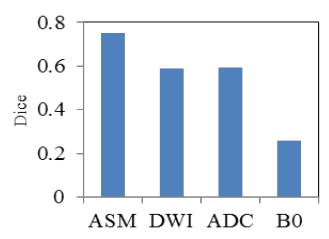

(a)

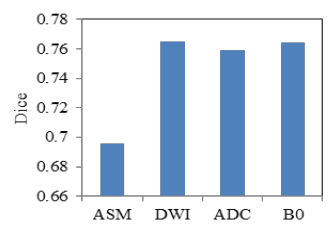

(b)

Figure 4. Contributions of different images: (a) Dice obtained from single image; (b) Dice obtained with one left out.

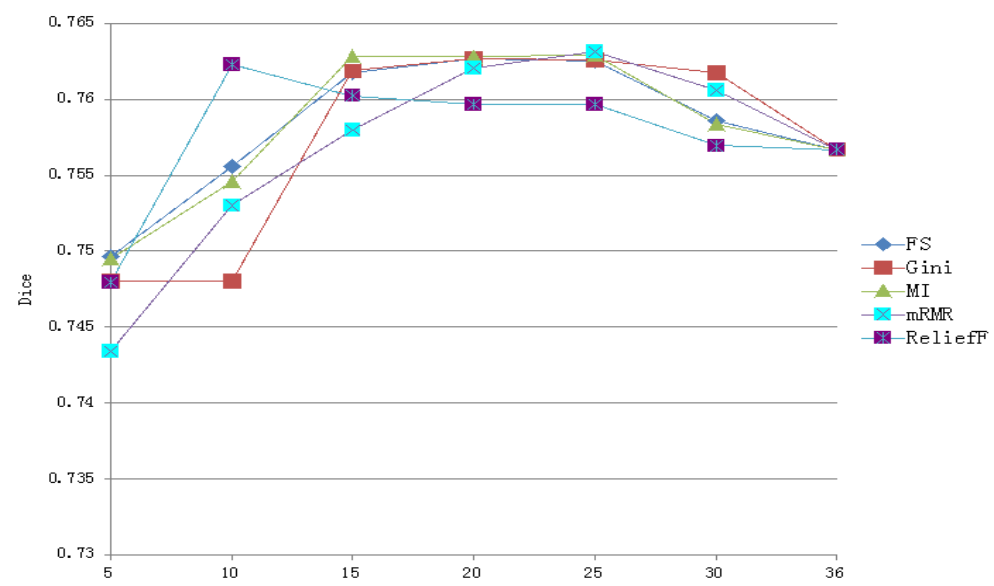

Figure 5. The Dice obtained from different feature selection algorithms: FS, Gini, MI, mRMR and ReliefF.

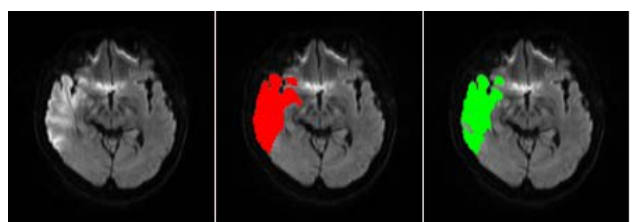

(a)

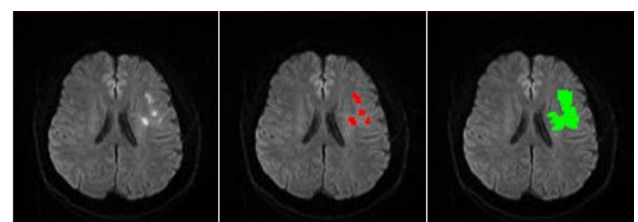

(b)

Figure 6. Segmentation of two DW axial slices with high (a) and low (b) Dice coefficient, red for ground truth infarcts and green for segmented infarcts. (a) Good segmentation; (b) Bad segmentation.

\section{Discussion}

In this paper, we have explored automated segmentation of hyper-acute infarcts from DW imaging based on carefully chosen features and SVM. As the DM method [3] is basically a global thresholding method to determine the DWI threshold based on divergence measure, it is not appropriate for processing data imaged within 6 hours due to the substantial variability in intensities. For the FCM based method [4], it is dependent on the prominent edge on DWI for confirmation of infarcts, which could fail because the DWI might have ill-defined edges. The proposed method could cope with the variability in intensities and ill-defined edges of DWI and ADC data imaged within 6 hours through learning the pattern from training samples. As such, the proposed method attain significantly higher accuracy in terms of Dice and sensitivity all with $p<0.001$.

From Figure 4, it can be seen that the classifier from ASM could yield the best Dice (0.749 \pm 0.122$)$ which is close to the Dice based the four images $(0.763 \pm 0.121)$. This may suggest that ASM has integrated the most relevant information from DWI, ADC and B0. On the other hand, the classifiers from DWI or ADC achieve a similar Dice (0.588 \pm 0.244 and $0.592 \pm 0.179$ respectively), which may suggest that both DWI and ADC alone could not achieve the optimum segmentation and they could provide complementary information to enhance infarct segmentation. Classifier based on B0 could only obtain a very low Dice $(0.256 \pm 0.213)$, which is consistent to the consensus that B0 carries little information on hyper-acute infarcts. From Figure 5, it can be seen that 
the best Dice is not achieved with the full features, indicating there are feature redundancy in the original 36 features. The best Dice is achieved with 15 features chosen from MI feature selection method.

To conclude, segmentation of hyper-acute ischemic infarcts based on SVM has achieved good results and features chosen from MI could yield an average Dice of $0.763 \pm 0.121$, which is significantly higher than existing methods. The proposed method could be a potential tool for clinicians to quantify the hyper-acute ischemic stroke and assist the decision making especially for thrombolytic therapy.

\section{Acknowledgements}

This work has been supported by: National Program on Key Basic Research Project (Nos. 2013CB733800 and 2013CB733803), National Science and Technology Pillar Program during the Twelfth Five-year Plan Period (No. 2011BAI08B09), Shenzhen Key Technical Development Grant (No. CXZZ20140610151856719), and Shenzhen Basic Research Grant (No. JCYJ20140414170821262). Authors would like to thank Dr. Yiqun Zhang for her valuable discussion on manually delineating infarct regions without clear boundaries.

\section{References}

[1] Du Plooy, J.N., Buys, A., Duim, W. and Pretorius, E. (2013) Comparison of Platelet Ultrastructure and Elastic Properties in Thrombo-Embolic Ischemic Stroke and Smoking Using Atomic Force and Scanning Electron Microscopy. Plos One, 8. http://dx.doi.org/10.1371/journal.pone.0069774

[2] Muir, K.W., Buchan, A., von Kummer, R., Rother, J. and Baron, J.C. (2006) Imaging of Acute Stroke. Lancet Neurol, 5, 755-768. http://dx.doi.org/10.1016/S1474-4422(06)70545-2

[3] Prakash, K.N.B., Gupta, V., Hu, J.B. and Nowinski, W.L. (2008) Automatic Processing of Diffusion-Weighted Ischemic Stroke Images Based on Divergence Measures: Slice and Hemisphere Identification, and Stroke Region Segmentation. Int J Comput Ass Rad, 3, 559-570.

[4] Tsai, J.Z., Peng, S.J., Chen, Y.W., Wang, K.W., Wu, H.K., Lin, Y.Y., Lee, Y.Y., Chen, C.J., Lin, H.J., Smith, E.E., Yeh, P.S. and Hsin, Y.L. (2014) Automatic Detection and Quantification of Acute Cerebral Infarct by Fuzzy Clustering and Histographic Characterization on Diffusion Weighted Mr Imaging and Apparent Diffusion Coefficient Map. Biomed Res Int, 13. http://dx.doi.org/10.1155/2014/963032

[5] Ma, L., Gao, P.Y., Hu, Q.M., Lin, Y., Jing, L.N., Xue, J., Wang, X.C., Chen, Z.J., Wang, Y.L., Liao, X.L., Liu, M.L. and Chen, W.J. (2010) Prediction of Infarct Core and Salvageable Ischemic Tissue Volumes by Analyzing Apparent Diffusion Coefficient without Intravenous Contrast Material. Acad Radiol, 17, 1506-1517. http://dx.doi.org/10.1016/j.acra.2010.07.010

[6] Hu, Q.M. and Nowinski, W.L. (2003) A Rapid Algorithm for Robust and Automatic Extraction of the Midsagittal Plane of the Human Cerebrum from Neuroimages Based on Local Symmetry and Outlier Removal. NeuroImage, 20, 2153-2165. http://dx.doi.org/10.1016/j.neuroimage.2003.08.009

[7] Cortes, C. and Vapnik, V. (1995) Support-Vector Networks. Mach. Learn., 20, 273-297. http://dx.doi.org/10.1007/BF00994018

[8] Chang, C.C. and Lin, C.J. (2011) LIBSVM: A Library for Support Vector Machines. Acm T Intel Syst Tec, 2.

[9] Cao, L., Li, J., Sun, Y., Zhu, H. and Yan, C. (2010) EEG-Based Vigilance Analysis by Using Fisher Score and PCA Algorithm. 2010 IEEE International Conference on Progress in Informatics and Computing (PIC), 175-179.

[10] Zhu, W. and Lin, Y. (2013) Using Gini-Index for Feature Weighting in Text Categorization. J Comput Inf Sys, 9, 58195826.

[11] Tourassi, G.D., Frederick, E.D., Markey, M.K. and Floyd, C.E. (2001) Application of the Mutual Information Criterion for Feature Selection in Computer-Aided Diagnosis. Med Phys, 28, 2394-2402. http://dx.doi.org/10.1118/1.1418724

[12] Peng, H., Long, F. and Ding, C. (2005) Feature Selection Based on Mutual Information: Criteria of Max-Dependency, Max-Relevance, and Min-Redundancy. IEEE Trans Pattern Anal Mach Intell, 27, 1226-1238. http://dx.doi.org/10.1109/TPAMI.2005.159

[13] Robnik-Sikonja, M. and Kononenko, I. (2003) Theoretical and Empirical Analysis of ReliefF and RReliefF. Mach. Learn., 53, 23-69. http://dx.doi.org/10.1023/A:1025667309714 